\title{
Efficient induction of differentiation and growth inhibition in IDH1 mutant glioma cells by the DNMT Inhibitor Decitabine.
}

\author{
Sevin Turcan ${ }^{1, *}$, Armida W. M. Fabius ${ }^{1, *}$, Alexandra Borodovsky ${ }^{2}$, Alicia Pedraza ${ }^{1}$, \\ Cameron Brennan ${ }^{1}$, Jason Huse ${ }^{1}$, Agnes Viale ${ }^{3}$, Gregory J. Riggins ${ }^{2}$, and Timothy \\ A. Chan ${ }^{1,4,5}$ \\ ${ }^{1}$ Human Oncology and Pathogenesis Program, Memorial Sloan-Kettering Cancer Center, New York, NY, USA. \\ 2 Department of Neurosurgery, School of Medicine, Johns Hopkins University, Baltimore, MD. \\ ${ }^{3}$ Genomics Core, Memorial Sloan-Kettering Cancer Center, New York, New York, USA. \\ ${ }^{4}$ Dept. of Radiation Oncology, Memorial Sloan-Kettering Cancer Center, New York, New York, USA. \\ ${ }^{5}$ Brain Tumor Center, Memorial Sloan-Kettering Cancer Center, New York, New York, USA. \\ * These authors contributed equally to this work \\ Correspondence to: Timothy A. Chan, email: chant@mskcc.org \\ Keywords: glioma, isocitrate dehydrogenase, methylation, epigenetics, treatment \\ Received: September 12, 2013 Accepted: September 16, $2013 \quad$ Published: September 16, 2013
}

This is an open-access article distributed under the terms of the Creative Commons Attribution License, which permits unrestricted use, distribution, and reproduction in any medium, provided the original author and source are credited.

\section{ABSTRACT:}

Mutation in the IDH1 or IDH2 genes occurs frequently in gliomas and other human malignancies. In intermediate grade gliomas, IDH1 mutation is found in over $70 \%$ of tumors. These mutations impart the mutant IDH enzyme with a neomorphic activity - the ability to synthesize 2-hydroxyglutarate (2-HG). This ability leads to a reprogramming of chromatin state, a block in differentiation, and the establishment of the glioma hypermethylator phenotype (G-CIMP). It has been hypothesized but not proven that the extensive DNA methylation that occurs in G-CIMP tumors helps maintain and "lock in" glioma cancer cells in a dedifferentiated state. Here, we tested this hypothesis by treating patient derived IDH1 mutant glioma initiating cells (GIC) with non-cytotoxic, epigenetically targeted doses of the DNMT inhibitor decitabine. Global methylome analysis of treated IDH1 mutant GICs showed that DAC treatment resulted in reversal of DNA methylation marks induced by IDH and the re-expression of genes associated with differentiation. Accordingly, treatment of IDH1 mutant glioma cells resulted in a dramatic loss of stem-like properties and efficient adoption of markers of differentiation, effects not seen in decitabine treated IDH wild-type GICs. Induction of differentiation was much more efficient than that seen following treatment with a specific inhibitor of mutant IDH enzyme (Agios). Decitabine also decreased replicative potential and tumor growth in vivo. Reexpression of polycomb regulated genes accompanied these DAC-induced phenotypes. In total, our data indicates that targeting the pathologic DNA methylation in IDH mutant cells can reverse mutant IDH induced hypermethylation and block in differentiation and promote tumor control. These findings have substantial impact for exploring new treatment strategies for patients with IDH mutant gliomas.

\section{INTRODUCTION}

Mutations in the metabolic enzyme isocitrate dehydrogenase 1 (IDH1) are found in $>70 \%$ intermediate grade gliomas [1,2], a disease which eventually progresses to high-grade glioma within 10 years. These mutations confer gain-of-function activity that allows the production of $(R)$-2-hydroxyglutarate $(2-\mathrm{HG})[3,4]$, a metabolite that is normally present at trace levels. Accumulation of 2-HG competitively inhibits various $\alpha$-ketoglutaratedependent dioxygenases [5]. IDH1 mutation functions by directly remodeling the epigenome to establish the glioma 
CpG island methylator phenotype (G-CIMP), inhibiting histone lysine demethylases and causing a block to cellular differentiation [6-9].

It has been hypothesized that the extensive DNA methylation that occurs in G-CIMP tumors maintains glioma cancer cells in a dedifferentiated state. The aberrant gene expression profile activated by mutant IDH1 confers a block to differentiation causing the malignant expansion of tumor-initiating cells with capacity to self-renew $[6,8]$. These findings raise the possibility that erasing the aberrantly hypermethylated marks may reverse the differentiation block induced by mutant IDH1. To explore this therapeutic possibility, we used the DNA demethylating agent, decitabine, a Food and Drug Administration (FDA) approved drug, to treat patient derived glioma tumor cells. We analyzed the effects of decitabine on both GICs with and without an endogeneous IDH1 mutation. IDH1-mutant GIC has been described previously [10].

The cytosine analogue 5-aza-2'-deoxycytidine (decitabine, DAC) is a hypomethylating agent used as a treatment for myelodysplastic syndrome. DAC exerts its effect by depletion and degradation of the maintenance DNA methyltransferase, DNMT1. Exposure to DNA demethylating agents is associated with altered hematopoietic differentiation and results in terminal differentiation of leukemia cells $[11,12]$. Further, DAC has the ability to cross the blood-brain barrier - the level of DAC attained in the cerebrospinal fluid can reach as high as half of its plasma concentration after a continuous intravenous infusion [13], making this drug an attractive therapeutic option for the management of gliomas. Recent studies have shown the efficacy of using low, epigenetically targeted doses of DNA demethylating agents in producing an antitumor memory response in both leukemic and epithelial tumors, including inhibition of subpopulations of cancer stem-cell like cells [14].

Although the impact of targeting the mutant enzyme with an IDH1 specific inhibitor has been evaluated [10], the effect was modest and did not lead to tumor regression. The efficacy of using DNA demethylating agents to treat mutant IDH1 expressing glioma cells has yet to be tested. Our results indicate that transient low doses of decitabine increases expression of genes associated with glial-astrocytic differentiation and induces differentiation in patient-derived IDH1-mutant tumor spheres. These findings begin to explore the efficacy of using an FDA approved drug in the management of IDH mutant gliomas.

\section{RESULTS}

\section{DAC induces differentiation of mutant IDH1 expressing glioma cells}

To study the effect of DAC on mutant IDH1 expressing gliomas, we utilized glioma tumor spheres
A

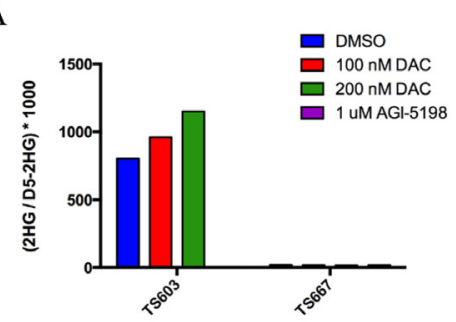

$\mathrm{C}$

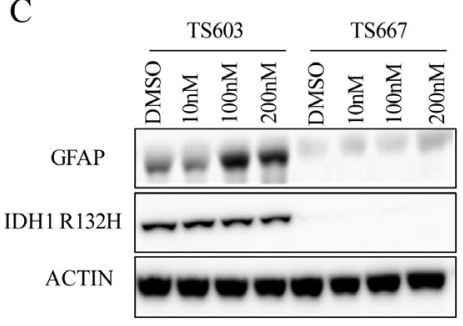

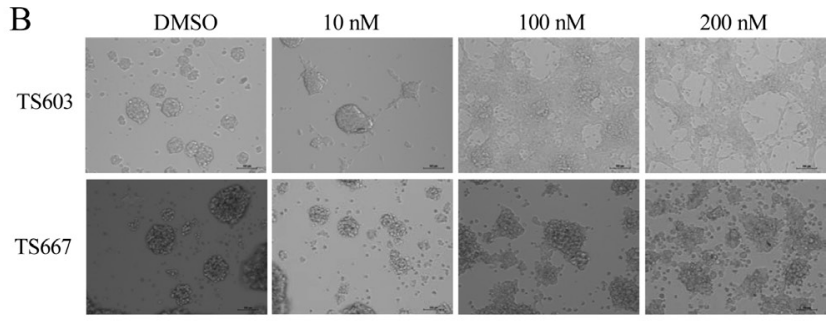

$\mathrm{D}$

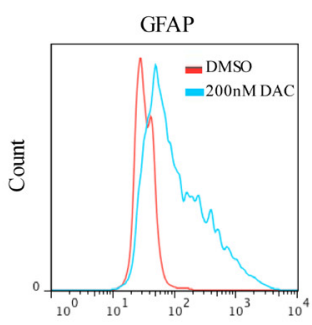

$\mathrm{E}$

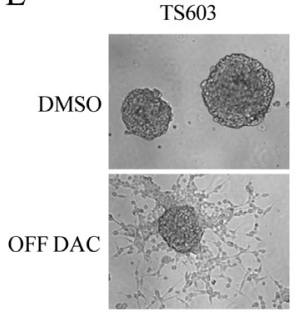

Figure 1: Decitabine efficiently induces differentiation in IDH1 mutant patient derived glioma initiating cells. A, DAC does not decrease 2HG levels. TS603 (IDH1 mutant) and TS667 (IDH wild-type) cells were treated as shown. For comparison, the mutant IDH1 inhibitor AGI-5198 was used in parallel, which dramatically lowered 2-HG levels in the TS603 line. B, DAC induces a differentiated morphology. Cells were treated with the indicated concentrations of DAC and bright-field images were taken at 10X magnification. At 200nM DAC, TS603 cells were adherent while the TS667 cells remained non-adherent spheres. C, DAC induces GFAP in TS603 cells but not in TS667 cells. Results from western blot with the indicated antibodies are shown. D, Flow cytometry results showing induction of GFAP protein levels in DAC treated IDH1 mutant TS603 cells. E, TS603 cells retain an adherent phenotype after withdrawal of DAC. Cells were treated with 200nM DAC for 7 days and then drug was removed and the cells were cultured for 3 weeks. 
that carry an endogenous heterozygous $\mathrm{R} 132 \mathrm{H}$ mutation WHO grade III anaplastic oligodendroglioma and harbor a co-deletion of $1 p$ and 19q. TS603 exhibits the G-CIMP phenotype and produces high $2 \mathrm{HG}$ levels in vitro [10]. As a control, we used the IDH wild-type oligogendroglioma tumor sphere line TS667. We used DAC at a nanomolar range $(10,100$ and $200 \mathrm{nM})$ to treat TS603 and TS667 glioma cells. These levels are non-cytotoxic [14]. 2-HG levels were unchanged in pellets of TS603 glioma cells after 7 days of treatment (Fig. 1A). Strikingly, 3 days of continuous exposure to DAC led to dramatic changes in the morphology of TS603 cells. At the $200 \mathrm{nM}$ dose, treated TS603 cells exhibited a differentiated morphology and became adherent (Fig. 1B). Furthermore, the differentiation phenotype was dose dependent, and was observed even at $10 \mathrm{nM}$ DAC where some cells grew as adherent spheres with a few differentiated cells in between spheres (Fig. 1B). Vehicle treated TS603 and TS667 cells and DAC treated TS667 cells continued to grow strictly as non-adherent spheres in culture and did not differentiate, suggesting that the differentiation phenotype is IDH1 mutant specific.

Next, we assessed protein levels of GFAP, a marker for glial differentiation. GFAP protein expression was (TS603). These cells were derived from a patient with

markedly increased in TS603 cells after 3-day treatment with 100 or $200 \mathrm{nM}$ DAC compared to vehicle treated cells (Fig. 1C, D). We did not observe any increase in GFAP expression in IDH wild-type TS667 cells.

We sought to determine whether transient treatment with DAC resulted in a "memory" type response that has recently been shown for transient low doses of DNA demethylating agents in hematological and epithelial tumors [14]. To test this hypothesis, we treated TS603 for 7 days with $200 \mathrm{nM}$ DAC, followed by drug withdrawal and culture in drug-free media for 3 weeks. While DNMT1 protein levels quickly recovered, the differentiation phenotype was maintained (but did reverse slowly) and transiently treated cells continued to grow as adherent cells (Fig. 1E).

Taken together, these results indicate that decitabine is able to efficiently reverse the differentiation block induced by mutant IDH1.

\section{Low dose DAC markedly impairs growth of mutant IDH1 expressing glioma cells}

We found that both 3- and 7- day exposure to 200 $\mathrm{nM}$ DAC led to a significant decrease in colony formation

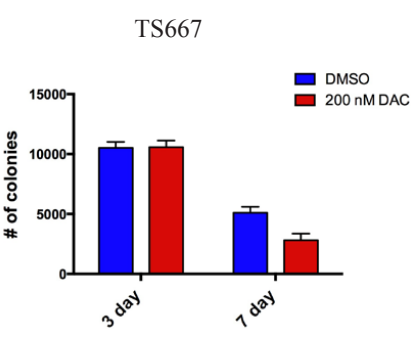

B
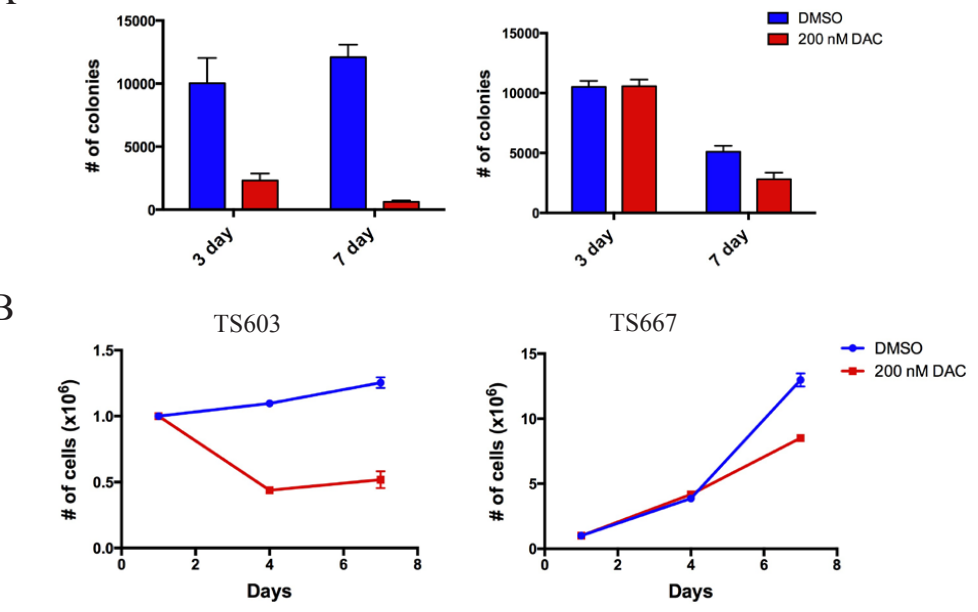

C

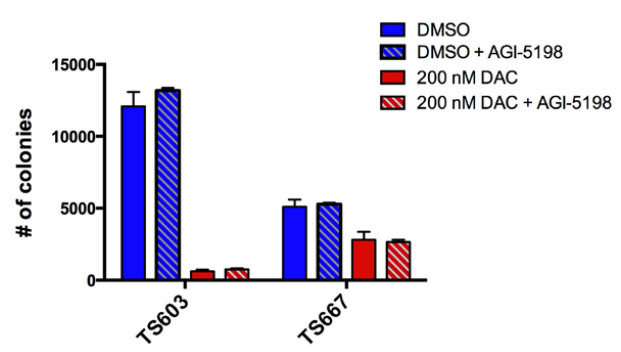

Figure 2: Low dose decitabine impairs growth potential in vitro and is superior to AGI-5198 in reducing proliferative capacity. A, Results from anchorage-independent growth assays using soft agar. Effect of DAC on IDH mutant TS603 and IDH wild-type TS667 cells. Cells were treated with 200nM DAC. All experiments done in triplicate. Data shown is mean $+/-1$ standard deviation. B, Cell growth curves showing that DAC treatment $(200 \mathrm{nM})$ decreases the proliferation of TS603. C, Effects of DAC (200nM) + AGI-5198 $(1 \mathrm{uM})$. The anti-proliferative effects observed with DAC alone were the same as with the combination. The growth suppressive effect was due entirely to DAC and not AGI-5198. 
ability of TS603 cells in soft agar, with $>90 \%$ reduction in colony formation ability occurring after 7-day exposure (Fig. 2A, left panel). In addition, cell growth was also suppressed by $60 \%$ in mutant IDH1 expressing TS603 after 3- and 7- days of $200 \mathrm{nM}$ DAC treatment (Fig. 2B, left panel). Although potent in the IDH mutant cells, the decrease in tumorigenicity was not entirely specific to TS603 cells. TS667 cells also showed decreased colony formation ability and cell growth, although the affect was not as dramatic and only occurred after 7 days of treatment with $200 \mathrm{nM}$ DAC. (Fig. 2A-B, right panels)

Next, we tested the efficacy of combining DAC with AGI-5198, a mutant IDH1 specific inhibitor. AGI5198 is highly selective for R132H mutation and under near complete $2 \mathrm{HG}$ inhibition induces modest expression of differentiation-associated genes. This is accompanied by decreased proliferation of IDH1-mutant glioma cells, without causing significant changes in genome-wide DNA methylation levels [10]. The effects of AGI-5198 were subtle and tumor regression never occurred. Therefore, we hypothesized that pre-treatment with $200 \mathrm{nM}$ DAC may further 'sensitize' the IDH1-mutant glioma cells to the therapeutic effects of AGI-5198. TS603 and TS667 cells were treated for 7 days with $200 \mathrm{nM}$ DAC, followed by DAC removal and plating in soft agar, at which point the cells were either treated with $1 \mu \mathrm{M}$ of AGI-5198 or DMSO continuously for 3 weeks. Pre-treatment with DAC did not allow the TS603 cells to respond more effectively to AGI-5198 (Fig. 2C). The numbers of colonies in DAC pretreated cells were similar between inhibitor and DMSO treated TS603 glioma cells, indicating that the dramatic reduction in anchorange-independent growth can be attributed to DAC alone. AGI-5198 did not cause additional loss of anchorage-independent growth.

A

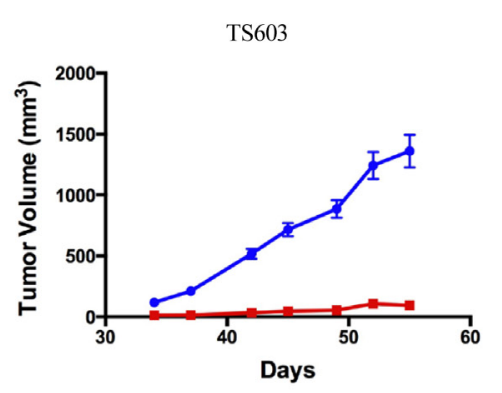

B

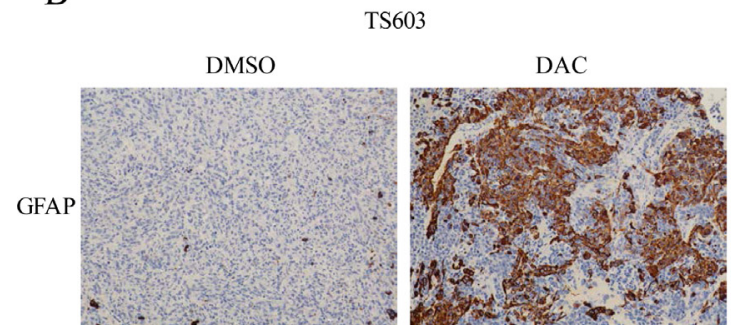

To test the growth inhibitory effects of DAC in vivo, we treated TS603 and TS667 cells with $200 \mathrm{nM}$ DAC for 7 days in vitro. We injected DAC or vehicle treated cells into the flanks of untreated SCID mice. Treatment with DAC markedly reduced the growth of TS603 xenografts by $>90 \%$. On the contrary, growth was more modestly impaired in the IDH1-wild-type TS667 glioma xenografts when compared to the IDH1-mutant expressing TS603 glioma xenografts (Fig. 3A). DAC treatment induced strong GFAP expression in IDH mutant TS603 cells but not in IDH wild-type TS667 cells (Fig. 3B).

\section{Low dose DAC inhibits genome-wide methylation and induces expression of genes associated with glial differentiation}

We evaluated the biochemical efficacy of DAC at low doses by assessing DNMT1 protein depletion after treatment. At the doses used, no cell death occurred. Both 3- and 7-day transient treatments with 100 and 200 nM DAC depleted DNMT1 levels, whereas $10 \mathrm{nM}$ dose resulted in diminished levels of DNMT1 in TS603 and TS667 glioma cells (Fig. 4A). Maximal depletion of DNMT1 was rapid and occurred as early as following a 3-day treatment.

To assess genome-wide changes in methylation levels, we treated TS603 and TS667 glioma cells with vehicle, $100 \mathrm{nM}$ DAC, $200 \mathrm{nM}$ DAC or $1 \mu \mathrm{M}$ AGI-5198 continuously for 2 weeks. DNA methylation was assessed using the Illumina Infinium HumanMethylation450 BeadChip. Overall, there was a significant reduction in the number of methylated loci in both TS603 and TS667 glioma cells, although the degree of hypomethylation
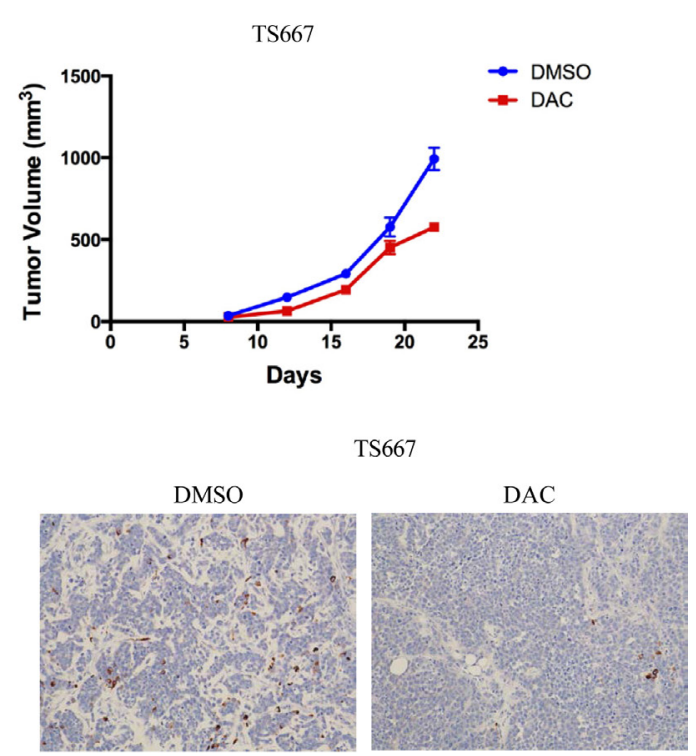

Figure 3: DAC suppresses growth and promotes differentiation of IDH mutant glioma cells. A, Xenograft assays showing that DAC treatment reduced tumor growth in vivo. The effects of TS603 (left) were superior to that of TS667 (right). At least 10 mice (20 tumors) were used. B, DAC induces GFAP expression in vivo. Xenografts were sectioned and stained for GFAP. Results from vehicle or DAC treated tumors are shown. 
was more pronounced in the IDH1-wild-type TS667 line (Fig. 4B). After a 2-week exposure to $200 \mathrm{nM}$ DAC, 30,915 probes (corresponding to 9996 unique genes) were hypomethylated in IDH1 mutant TS603 glioma cells and 130,017 probes (corresponding to 17,182 unique genes) were hypomethylated in TS667 cells $(\Delta \beta$ value $<-0.4)$ (Supplemental Table 1). In contrast, only 52 probes were hypomethylated at $\Delta \beta<-0.4$ in TS603 line after a 2-week treatment with $1 \mu \mathrm{M}$ AGI-5198 (Supplemental Table 1). Therefore, low dose DAC was a potent inhibitor of DNMT1 and loss of DNA methylation.

In addition to genome-wide methylation, we also assessed gene expression changes using Affymetrix gene expression arrays following a 2-week treatment with DMSO, 100nM DAC, $200 \mathrm{nM}$ DAC or AGI-5198. In TS603 glioma cells, 411 unique genes were upregulated and 82 genes were downregulated after DAC treatment (Supplemental Table 2). Compared with vehicle-treated TS603 cells, low dose transient DAC treatment resulted in the upregulation of a number of genes associated with glial-astrocytic differentiation, such as GFAP and LGALS3 [15], whereas NEUROD1, a gene associated with neural stem cells, was downregulated (Fig. 4C). Some of the most significantly enriched pathways (BH-adjusted $p<0.05$ ) among the upregulated genes included ECM-receptor interaction, cytokine-cytokine receptor interaction and vasculature development (Supplemental Table 3).

When compared to vehicle treated cells, in IDH wild-type TS667 glioma cells, 385 unique genes were upregulated and 78 genes were downregulated after DAC treatment. Overall, 110 genes were upregulated in both cell lines after $200 \mathrm{nM}$ DAC treatment, and upregulation of GFAP and other glial differentiation markers was not evident in TS667 glioma cells (Supplemental Table 2).

We next integrated the DNA methylation and gene expression data to identify genes that increase their gene expression following loss of methylation. Compared to vehicle treated controls, $79 \%$ of all the upregulated genes in TS667 cells were hypomethylated. In TS603 glioma cells, $48 \%$ of all upregulated genes (197 genes) were also
A

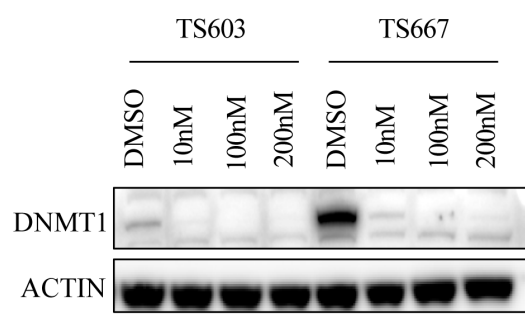

B

DMSO
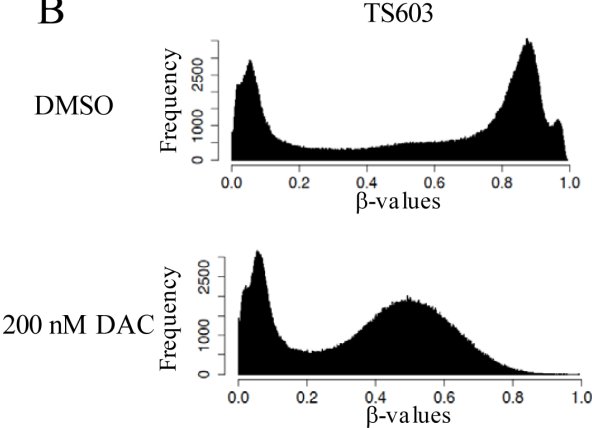

$\mathrm{D}$

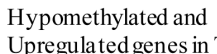

Hypomethylated and
Upregulated genes in

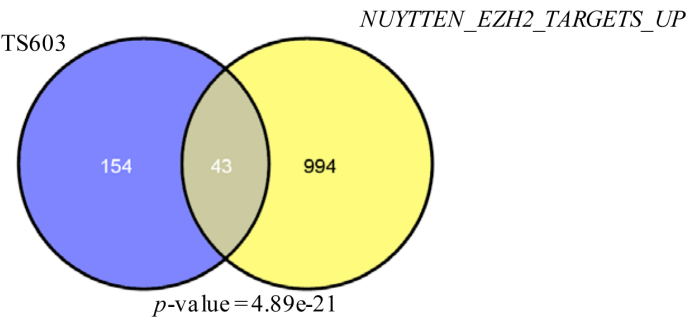

$\mathrm{C}$

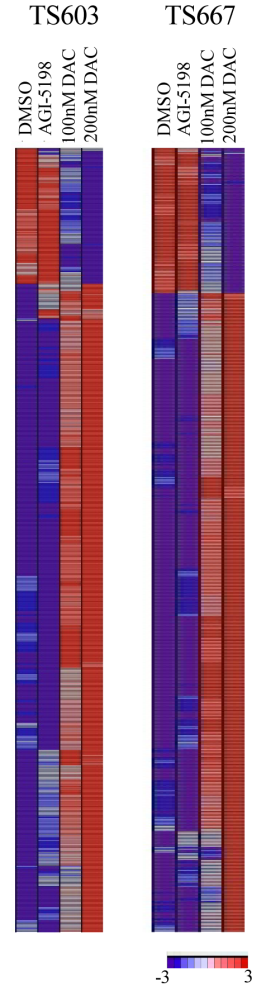

Figure 4: Decitabine reverses genome-wide DNA methylation and induces expression of genes associated with differentiation in IDH mutant glioma cells. A, Low dose DAC inhibits DNMT1. Results from western blot shown. B, DAC treatment results in loss of DNA methylation. DNA methylome analysis of TS603 and TS667 cells following DAC treatment (200nM) is shown. Results from the Illumina HumanMethylation450 array. C, Gene expression changes following DAC treatment (200nM). Results from Affymetrix gene expression arrays. Most significantly altered genes following 200nM DAC are shown. D, Significant concordance between demethylated and upregulated genes and polycomb targets. Venn diagram showing overlap between the gene sets. P value (hypergeometric) is shown. 
hypomethylated (Supplemental Table 4). Importantly, this group also included all the differentiation-associated genes. To gain further insight into the genes with increased expression following 200nM DAC treatment in TS603 cells, our gene set consisting of hypomethylated and upregulated genes were analyzed using the molecular signature database (MSigDB) and the degree of overlap with the $\mathrm{c} 2$ curated gene sets was calculated. These results showed highly significant enrichment of NUYTTEN EZH2 TARGETS UP, which includes genes up-regulated in PC3 cells following EZH2 knockdown [16], indicating that DAC treatment may erase methylation marks from Polycomb EZH2 marked loci and subsequently activate genes involved in differentiation (Fig. 4D). These data point to the re-expression of Polycomb controlled genes as playing an important role in how DAC reverses the effects of mutant IDH.

\section{DISCUSSION}

Tumor-initiating cells are thought to contribute to relapse or tumor recurrence after treatment, therefore a major challenge is to discover agents or treatment paradigms that will target this stem-cell like subpopulation. Our findings demonstrate the efficacy of DNA demethylating agents in targeting gliomainitiating cells and highlight the differential response of IDH1 mutant versus IDH1 wild-type glioma cells to low doses of DAC. Transient exposure to nanomolar concentrations of DAC induces the differentiation of the IDH1 R132H, but not IDH1 wild-type glioma-initiating cells. Importantly, exposure to nanomolar doses of DAC has long-lasting effects on continued differentiation, long after the demethylating agent is removed and DNMT1 levels are restored. This may be explained by the fact that exposure to DNA demethylating agents removes the DNA methylation marks at the promoters of glial differentiation genes such as GFAP and may also relax the formation of heterochromatin at the promoters of differentiation genes leading to their upregulation. In conjunction with increased differentiation in IDH1 R132H glioma cells, DAC also exerts its antitumor effects by reducing the tumorigenic potential of IDH1-mutant cells by decreasing colony formation in vitro and xenograft growth in vivo. It remains to be seen whether DNA demethylating agents can sensitize glioma-initiating cells to other cytotoxic or targeted drugs, as has been suggested in lung and ovarian cancers $[17,18]$. In our initial studies, we have not observed sensitization of IDH1-mutant glioma cells to IDH1 R132H specific inhibitor, AGI-5198 following DAC exposure; however, further studies are needed.

Our studies also indicate that growth of IDH1 wildtype glioma cells are reduced following transient treatment with DAC, albeit through alternate pathways. The distinct gene expression program and the lack of differentiation phenotype observed in IDH1-wild-type cells suggest a different mechanism of action induced by DAC. Given the vast heterogeneity of glioma, a broader investigation of the differential therapeutic responses of glioma subgroups to transient low doses of DNA demethylating agents will shed light onto the therapeutic benefit of these agents as either mono- or combination therapies. Importantly, a companion paper by Greg Riggin's group at Johns Hopkins shows analygous results using a separately derived IDH mutant GIC line [19]. Together, our studies highlight the therapeutic potential of DNMT inhibitors for treatment of IDH mutant tumors.

The use of DAC to target mutant IDH dependent pathobiology is tantalizing. DAC is already FDA approved and penetrates the CNS very well. In our preclinical models, we show that using DAC can effectively reverse the pathologic DNA methylation induced by mutant IDH. Based on our data, we hypothesize that DAC may be useful for other tumor types, such as leukemias and chondrosarcomas, which have IDH mutation.

In summary, our findings highlight the utility of DNA demethylating agents in the management of glioma, a highly malignant disease with dismal prognosis. The ability to induce a sustained differentiation phenotype in the tumor-initiating subpopulation of IDH1 R132H gliomas may provide a promising therapeutic window of opportunity for tumors with this mutation.

\section{METHODS}

\section{Generation of patient derived glioma-initiating cells and drug treatment.}

IDH1-R132H TS603 and IDH1-wild-type TS667 glioma spheres were derived from patients undergoing tumor resection at Memorial Sloan-Kettering Cancer Center (MSKCC). Tumors were obtained in accordance with Institutional Review Board policies at MSKCC. 10 nM, 100 nM , 200 nM Decitabine (Sigma-Aldrich, St. Louis, MO) ,1 $\mu \mathrm{M}$ of AGI-5198 IDH1 R132H specific inhibitor (Xcess Bio) or DMSO were added to the media. Decitabine was added every 24 hours with daily medium change and AGI-5198 was added every other day with medium change.

\section{Soft agar assays.}

Cells were treated with decitabine for 3- or 7- days before plating for soft agar. 100,000 cells were plated in complete Neurocult media with growth supplements (Stem Cell Technologies) into 6 -well plates containing $0.65 \%$ top and bottom agar. Cells were plated in the middle layer in complete Neurocult media containing $0.40 \%$ agar. Cells were treated with or without AGI-5198 for 3-4 weeks, and media was refreshed every 2 days. After 2-4 weeks, 
colonies were stained with $0.0005 \%$ crystal violet and quantified using a Gelcount colony counter (Oxford Optronix).

\section{Xenografts.}

TS603 and TS667 glioma cells were pretreated with $200 \mathrm{nM}$ decitabine or DMSO for 7 days. After harvesting, $1 \times 10^{6}$ cells were injected subcutaneously (100 $\mu$ l volume, equal parts Matrigel and media) into both flanks of 5-6 week old female SCID mice. Once tumors researched a palpable size, tumor size was measured every 3-4 days by a caliper. Protocols for all treatments were approved by the Institutional Animal Care and Use Committee and strict guidelines were enforced.

\section{Western blot.}

Cells were lysed in CelLytic (Sigma) with protease inhibitors (Roche). Proteins were separated by SDSPAGE, transferred to PVDF membrane (Millipore) and probed with the following primary antibodies: anti-IDH1 R132H (Dianova, DIA-H09), anti-DNMT1 (New England Biolabs, M0230S), anti-GFAP (Cell Signaling Tech, 2118S) and anti- $\beta$-actin (Sigma, A5316).

\section{Flow Cytometry.}

For single-color flow cytometry, $10^{6}$ cells were washed with ice-cold PBS, permeabilized and fixed using BD Cytoperm/Cytofix solution (BD, PharMingen), and incubated with anti-GFAP (1:200, BD Pharmingen) for $30 \mathrm{~min}$ at room temperature. Cells were washed with PBS and analyzed with FACScan flow cytometer (Becton Dickinson). Fluorescence-activated cell sorter data were analyzed using FLowJo Software (TreeStar).

\section{Immunohistochemistry.}

Paraffin-embedded sections of xenografts were deparaffinized. The sections were then stained with either hematoxylin and eosin (H\&E), Ki-67 or GFAP. Detection was performed with the DAB Map kit (Ventana Medical Systems).

\section{Sample preparation.}

DNA from DMSO, DAC or IDH1 inhibitor treated cells were extracted with the DNeasy Blood and Tissue Kit (Qiagen) and RNA was isolated with RNeasy Plus Mini Kit according to the manufacturer's directions.

\section{Genomic analysis.}

Expression analysis was performed using the Affymetrix U133 2.0 microarray (Affymetrix). Genomewide methylation analysis was performed using the Infinium HumanMethylation450 bead array (Illumina). Processing of the arrays was per the manufacturers' protocol. Methylation data were extracted using GenomeStudio software (Illumina). Methylation values for each site are expressed as a beta $(\beta)$ value, representing a continuous measurement from 0 (completely unmethylated) to 1 (completely methylated). This value is based on following calculation: $\beta$ value $=$ (signal intensity of methylation-detection probe)/(signal intensity of methylation- detection probe + signal intensity of nonmethylation detection).

\section{Data analysis.}

For methylation analysis, Illumina data were imported into Partek software. $\beta$ values for 200 nM DAC or 1uM AGI-5198 treated cells were substracted from $\beta$ values for DMSO treated cells. Any probe with $\Delta \beta<$ -0.4 were considered to be hypomethylated following treatment. For gene expression analysis, microarrays were RMA normalized and fold changes were calculated by subtracting the log intensity values of $200 \mathrm{nM}$ DAC or 1uM AGI-5198 from DMSO treated cells, and probes with absolute fold changes $>1.2$ were considered to be differentially expressed. DAVID was used to identify significantly overrepresented pathways [20]. For gene set analysis, hypomethylated and upregulated genes were input into the molecular signature database (MSigDB) and statistical significance of overlaps were calculated between our gene sets and the $\mathrm{C} 2$ (curated gene sets) library from MSigDB.

\section{2-HG analysis.}

2HG levels were determined by mass spectrometry as previously described [6].

\section{ACKNOWLEDGEMENTS}

We would like to thank the Memorial Sloan Kettering Brain Tumor Center for support. Sevin Turcan was supported by an NIH T32 grant (5T32CA160001). This work was supported by the Frederick Adler Fund (TAC), the Sontag Foundation (TAC), and NIH grant RO1CA154767 (TAC). We thank Justin Cross for excellent technical assistance with 2-HG measurements. 


\section{REFERENCES}

1. Yan H, Parsons DW, Jin G, McLendon R, Rasheed BA, Yuan W, Kos I, Batinic-Haberle I, Jones S, Riggins GJ, Friedman H, Friedman A, Reardon D, Herndon J, Kinzler $\mathrm{KW}$, Velculescu VE, et al. IDH1 and IDH2 mutations in gliomas. The New England journal of medicine. 2009; 360(8):765-773.

2. Parsons DW, Jones S, Zhang X, Lin JC, Leary RJ, Angenendt P, Mankoo P, Carter H, Siu IM, Gallia GL, Olivi A, McLendon R, Rasheed BA, Keir S, Nikolskaya T, Nikolsky Y, et al. An integrated genomic analysis of human glioblastoma multiforme. Science. 2008; 321(5897):18071812.

3. Dang L, White DW, Gross S, Bennett BD, Bittinger MA, Driggers EM, Fantin VR, Jang HG, Jin S, Keenan MC, Marks KM, Prins RM, Ward PS, Yen KE, Liau LM, Rabinowitz JD, et al. Cancer-associated IDH1 mutations produce 2-hydroxyglutarate. Nature. 2009; 462(7274):739744.

4. Ward PS, Patel J, Wise DR, Abdel-Wahab O, Bennett BD, Coller HA, Cross JR, Fantin VR, Hedvat CV, Perl AE, Rabinowitz JD, Carroll M, Su SM, Sharp KA, Levine $\mathrm{RL}$ and Thompson CB. The common feature of leukemiaassociated IDH1 and IDH2 mutations is a neomorphic enzyme activity converting alpha-ketoglutarate to 2-hydroxyglutarate. Cancer cell. 2010; 17(3):225-234.

5. Xu W, Yang H, Liu Y, Yang Y, Wang P, Kim SH, Ito S, Yang C, Wang P, Xiao MT, Liu LX, Jiang WQ, Liu J, Zhang JY, Wang B, Frye S, et al. Oncometabolite 2-hydroxyglutarate is a competitive inhibitor of alphaketoglutarate-dependent dioxygenases. Cancer cell. 2011; 19(1):17-30

6. Turcan S, Rohle D, Goenka A, Walsh LA, Fang F, Yilmaz E, Campos C, Fabius AW, Lu C, Ward PS, Thompson CB, Kaufman A, Guryanova O, Levine R, Heguy A, Viale A, et al. IDH1 mutation is sufficient to establish the glioma hypermethylator phenotype. Nature. 2012; 483(7390):479483.

7. Lu C, Ward PS, Kapoor GS, Rohle D, Turcan S, AbdelWahab O, Edwards CR, Khanin R, Figueroa ME, Melnick A, Wellen KE, O'Rourke DM, Berger SL, Chan TA, Levine RL, Mellinghoff IK, et al. IDH mutation impairs histone demethylation and results in a block to cell differentiation. Nature. 2012; 483(7390):474-478.

8. Duncan CG, Barwick BG, Jin G, Rago C, Kapoor-Vazirani P, Powell DR, Chi JT, Bigner DD, Vertino PM and Yan H. A heterozygous IDH1R132H/WT mutation induces genome-wide alterations in DNA methylation. Genome research. 2012; 22(12):2339-2355.

9. Figueroa ME, Abdel-Wahab O, Lu C, Ward PS, Patel J, Shih A, Li Y, Bhagwat N, Vasanthakumar A, Fernandez HF, Tallman MS, Sun Z, Wolniak K, Peeters JK, Liu W, Choe SE, et al. Leukemic IDH1 and IDH2 mutations result in a hypermethylation phenotype, disrupt TET2 function, and impair hematopoietic differentiation. Cancer cell. 2010; 18(6):553-567.

10. Rohle D, Popovici-Muller J, Palaskas N, Turcan S, Grommes C, Campos C, Tsoi J, Clark O, Oldrini B, Komisopoulou E, Kunii K, Pedraza A, Schalm S, Silverman L, Miller A, Wang F, et al. An inhibitor of mutant IDH1 delays growth and promotes differentiation of glioma cells. Science. 2013; 340(6132):626-630.

11. Jones PA and Taylor SM. Cellular differentiation, cytidine analogs and DNA methylation. Cell. 1980; 20(1):85-93.

12. Pinto A, Attadia V, Fusco A, Ferrara F, Spada OA and Di Fiore PP. 5-Aza-2'-deoxycytidine induces terminal differentiation of leukemic blasts from patients with acute myeloid leukemias. Blood. 1984; 64(4):922-929.

13. Chabot GG, Rivard GE and Momparler RL. Plasma and cerebrospinal fluid pharmacokinetics of 5-Aza-2'deoxycytidine in rabbits and dogs. Cancer research. 1983; 43(2):592-597.

14. Tsai HC, Li H, Van Neste L, Cai Y, Robert C, Rassool FV, Shin JJ, Harbom KM, Beaty R, Pappou E, Harris J, Yen RW, Ahuja N, Brock MV, Stearns V, Feller-Kopman D, et al. Transient low doses of DNA-demethylating agents exert durable antitumor effects on hematological and epithelial tumor cells. Cancer cell. 2012; 21(3):430-446.

15. Yang JW, Kang SU, Engidawork E, Rodrigo R, Felipo V and Lubec G. Mass spectrometrical analysis of galectin proteins in primary rat cerebellar astrocytes. Neurochemical research. 2006; 31(7):945-955.

16. Nuytten M, Beke L, Van Eynde A, Ceulemans H, Beullens M, Van Hummelen P, Fuks F and Bollen M. The transcriptional repressor NIPP1 is an essential player in EZH2-mediated gene silencing. Oncogene. 2008; 27(10):1449-1460.

17. Juergens RA, Wrangle J, Vendetti FP, Murphy SC, Zhao M, Coleman B, Sebree R, Rodgers K, Hooker CM, Franco N, Lee B, Tsai S, Delgado IE, Rudek MA, Belinsky SA, Herman JG, et al. Combination epigenetic therapy has efficacy in patients with refractory advanced non-small cell lung cancer. Cancer discovery. 2011; 1(7):598-607.

18. Matei D, Fang F, Shen C, Schilder J, Arnold A, Zeng $\mathrm{Y}$, Berry WA, Huang $\mathrm{T}$ and Nephew KP. Epigenetic resensitization to platinum in ovarian cancer. Cancer research. 2012; 72(9):2197-2205.

19. Borodovsky A, Salmasi V, Turcan S, Baia G, Eberhart C, Weingart J, Gallia G, Baylin S, Chan T and Riggins G. 5 -azacytidine reduces methylation, promotes differentiation and induces tumor regression in a patient-derived IDH1 mutant glioma xenograft. Oncotarget. 2013.

20. Huang da W, Sherman BT and Lempicki RA. Systematic and integrative analysis of large gene lists using DAVID bioinformatics resources. Nature protocols. 2009; 4(1):4457. 